\title{
Language-Specific Dysgraphia in Persian Patients with Right Brain Stroke: Influence of Visuospatial Neglect and Rehabilitation
}

\author{
Shole Vatanparasti (iD ${ }^{1}$, Anoshirvan Kazemnejad (iD) ${ }^{2}$, Shahram Oveisgharan (iD) ${ }^{3}$, Payman Hassani \\ Abharian ${ }^{4}$ and Ali Yoonessi (ii) ${ }^{5, *}$ \\ ${ }^{1}$ Institute for Cognitive Sciences Studies, Tehran, Iran \\ ${ }^{2}$ Department of Biostatistics, Faculty of Medical Sciencess, Tarbiat Modares University, Tehran, Iran \\ ${ }^{3}$ Rush Alzheimer's Disease Center, Rush University Medical Center, Chicago, IL, United States \\ ${ }^{4}$ Department of Rehabilitation, Institute for Cognitive Science Studies, Tehran, Iran \\ ${ }^{5}$ Neuroscience Department, School of Advanced Technologies in Medicine, Tehran University of Medical Sciences, Tehran, Iran \\ "Corresponding author: Neuroscience Department, School of Advanced Technologies in Medicine, Tehran University of Medical Sciences, \#88 Italy Ave., Tehran, Iran. Tel: \\ +98-2188991106, Fax: +98-2188991117 Email: yoonessi@gmail.com
}

Received 2019 March 09; Revised 2019 May 18; Accepted 2019 May 29.

\begin{abstract}
Background: Writing is a human communication tool. No disability behavioral assessment of dysgraphia due to stroke-induced neglect has been conducted in Iran.

Objectives: We investigated rehabilitation of dysgraphia in patients with stroke-related neglect in Persian language- specific constructive errors of writing.

Methods: All patients were suffering from stroke-induced visual neglect. Five patients were evaluated for dysgraphia in a spontaneous writing task before and after rehabilitation without writing practice for 10 sessions with prism adaption (PA). Dysgraphia was classified into visuospatial omission error, visuospatial destruction error, visuospatial size error, visuospatial tilting error, poor handwriting style, visuospatial distance error, and visuospatial perseveration (addition) error. Neglect was evaluated using the star cancellation test (SCT), and line bisection test (LBT) and activities of daily living (ADL) was assessed by the Barthel index (BI) and the Catherine Bergego scale (CBS).

Results: All patients showed significant improvement in dysgraphia (measured using spontaneous writing test), ADL (measured using CBS and BI), and neglect (measured using LBT and SCT $)(\mathrm{P}<0.05)$. Visuospatial destruction errors were the most frequent and visuospatial omission errors were the least frequent errors.

Conclusions: PA is a potential effective strategy in stroke rehabilitation of dysgraphia, visuospatial neglect and ADL. Unilateral spatial neglect and rehabilitation improves dysgraphia in Persian language speaking patients with right brain stroke.
\end{abstract}

Keywords: Rehabilitation, Neglect, Dysgraphia, Writing, Language, Prism Adaption

\section{Background}

Dysgraphia may be caused by acute right parietal infarctions. Lesions in the right hemisphere of the brain often cause a non-aphasic disorder known as spatial dysgraphia (1-6). Dysgraphia, also known as spatial writing disorders, is defined by inscribing errors which commonly contain missing elements, omission errors, addition errors, tilting error, visuospatial destruction mistake, and syllabic tilting fault. These errors have been observed in patients of various languages, such as Japanese (3), English (7), French (8), Spanish (4), and Korean, with right-brain stroke (9-11). However, writing disorder due to right-brain stroke has various forms associated with the specific writing system of a given language. For example, Japanese pa- tients with right-brain stroke demonstrated disorders related to ideogram (Kanji) writing but not to phonograms (Kana) (3).

However, these language-specific dysgraphia or constructive errors of writing are not quite understood. The errors may be affected by visuospatial processing, have a convened array of letters of the alphabet, and require a particular rehabilitation strategy of their letters of the alphabet graphemic systems (3, 9, 10, 12-15). Stroke-induced neglect is an important factor to influence dysgraphia and is usually a comorbid sign. In a recent study, Prism adaptation (PA) as a single therapeutic approach that has been shown to be effective on neglect and dysgraphia following stroke (8). 
The Persian alphabet, Farsi, includes 32 letters of the alphabet, 9 vowels and 31 consonants. Each Persian grapheme must be located within a square space to word form right side to left side for spatial structure. Accordingly, in the Persian writing system, dysgraphia and writing performance after a right-brain stroke may be exclusively affected if unilateral visuospatial neglect is also present.

\section{Objectives}

Nevertheless, no study to date has examined the relation between Persian language-specific dysgraphia (constructive errors of writing) and spatial neglect and rehabilitation. The present study detected a pattern of dysgraphia before intervention, and investigated rehabilitation with prism adaption to treat dysgraphia in patients with neglect.

\section{Methods}

This interventional, pilot study was conducted in the Stroke Unit of Shariati Hospital, Iran, from August 2017 to September 2018.

\subsection{Subjects}

A total of 5 subjects completed the treatment course and enrolled. All 5 participants in PA group were right handed; 4 (80\%) were men and 1 (20\%) were women with a mean 60 years. Education was between 9 and 16 years. They were tested for dysgraphia using spontaneous writing. They were also requested to attend 10 rehabilitation sessions of the PA. The inclusion criteria consisted of strokereduced neglect, dysgraphia, and completion of more than 5 years of grade school education, right-handedness, and right hemisphere stroke. The exclusion criteria were age less than 18 and more than 80 years, a previous history of a writing deficit, brain trauma, implanted heart pacemaker, cerebral edema, epilepsy and intense pain. In terms of type of stroke, 3 (60\%) participants had ischemic cerebral infarction compared to hemorrhagic and stroke onset date was within the last 6 months prior to randomization in 2 (40)\% of patients. Characteristics of participants are summarized in Table 1.

\subsection{Assessment of Writing and Measurement Technique}

All participants completed the spontaneous writing task. Patients made spontaneous writing error (dysgraphia) due to stroke-induced neglect start and finish intervention. The participants performed the task seated in a chair in a quiet environment. The cognitive neuropsychology therapist sat directly in front of the participant and presented him/her with the task material. No time limit was considered for the task. The spontaneous writing task consisted of writing their name, address, and autobiographical memory including where, what, and when on an A4-sized plain paper start and finish intervention. Only constructive writing errors were evaluated as dysgraphia, and semantic (linguistic) errors were not assessed. In most previous studies, constructive writing errors have been classified into tilting and visuospatial omission in accordance with the criteria determined by Yoon et al. (10). We used a new suggestion for the detection on classification of dysgraphia in the writing system. Constructive writing errors were further classified into visuospatial destruction error, visuospatial omission, visuospatial tilting, visuospatial poor handwriting, visuospatial size error, visuospatial distance error, and visuospatial perseveration errors (Figure 1). Visuospatial destruction errors are the creation of a nonexistent form. Visuospatial omission errors mean that the writer ignores more than $50 \%$ of space in the square and deletes 1 part of the grapheme. Visuospatial tilting errors occur when the lines of writing are not horizontal. Visuospatial size error means the writer changes normal size writing to large size writing. Visuospatial distance error is error in the distance between writing lines. Visuospatial perseveration error is related to the repetition and addition to the writing. Evaluation of writing and unilateral spatial neglect was conducted before and after treatment with PA.

3.3. Assessment of Unilateral Spatial Neglect and Functional Activity of Daily Living and Measurement Technique

All participants were evaluated using the star cancellation test (SCT) (16), and line bisection test (LBT) $(17,18)$ for visuospatial unilateral neglect and the Barthel index (BI) (19) and the Catherine Bergego scale (CBS) (20-22) for activities of daily living (ADL). Experimental group underwent PA therapy.

The SCT is scored through counting the number of small stars marked by the patients (a total of 100 small and large stars; 50 small stars) on an A4 paper. The number of stars marked was calculated on the left side of the page. A score between 0 and $46 \%$ illustrates unilateral visuospatial neglect.

In the LBT, patients were asked to bisect 40 horizontal lines at their middle (18 lines on the middle, 10 lines on the left side, and 12 lines on the right side) on an A4 paper. For each patient, we measured the absolute distance between the patient's bisection and the middle lines' points. 


\begin{tabular}{|c|c|c|c|c|c|c|c|}
\hline $\mathrm{N} /$ Intervention & Age, $y$ & Gender & $\begin{array}{l}\text { Grade School } \\
\text { Education }\end{array}$ & $\begin{array}{l}\text { Region of Stroke: P, } \\
\text { T, F, O, IN, TH }\end{array}$ & $\begin{array}{l}\text { Time Since the } \\
\text { Stroke Onset: The } \\
\text { Chronic/Sub-Acute }\end{array}$ & $\begin{array}{l}\text { Type of the Stroke: } \\
\text { I/H }\end{array}$ & Writing Errors \\
\hline 1- PA & 46 & Female & 16 & RTH, & S-A & $\mathrm{H}$ & Omission \\
\hline 2- PA & 63 & Male & 9 & $\mathrm{RO}, \mathrm{RP}$ & S-A & I & $\begin{array}{l}\text { Omission, } \\
\text { destruction, size, } \\
\text { distance, tilting, } \\
\text { poor handwriting }\end{array}$ \\
\hline 3- PA & 70 & Male & 16 & $\mathrm{RT}, \mathrm{RF}$ & $\mathrm{C}$ & I & Tilting \\
\hline 4- PA & 65 & Male & 12 & RT & $\mathrm{C}$ & I & $\begin{array}{l}\text { Omission, } \\
\text { destruction, size, } \\
\text { distance, tilting }\end{array}$ \\
\hline 5- PA & 67 & Male & 16 & $\mathrm{RT}, \mathrm{RF}, \mathrm{RP}, \mathrm{TH}$ & $\mathrm{C}$ & $\mathrm{H}$ & $\begin{array}{l}\text { Destruction, } \\
\text { perseveration, size. } \\
\text { tilting, poor } \\
\text { handwriting }\end{array}$ \\
\hline $\mathrm{PA}$, mean $\pm \mathrm{SD}$ & $60 \pm 10$ & & $14.2 \pm 2.48$ & & & & $\begin{array}{l}\text { Pre } 8(2.40) \text { vs. post } \\
1.2(0.80)\end{array}$ \\
\hline
\end{tabular}

${ }^{\mathrm{z}}$ Abbreviations: C, chronic; F, cortex frontal; H, intracranial hemorrhage; I, intracranial ischemic; P, cortex parietal; PA, prism adaptation; R, right; S-A, sub-acute; T, cortex temporal; TH, thalamus.
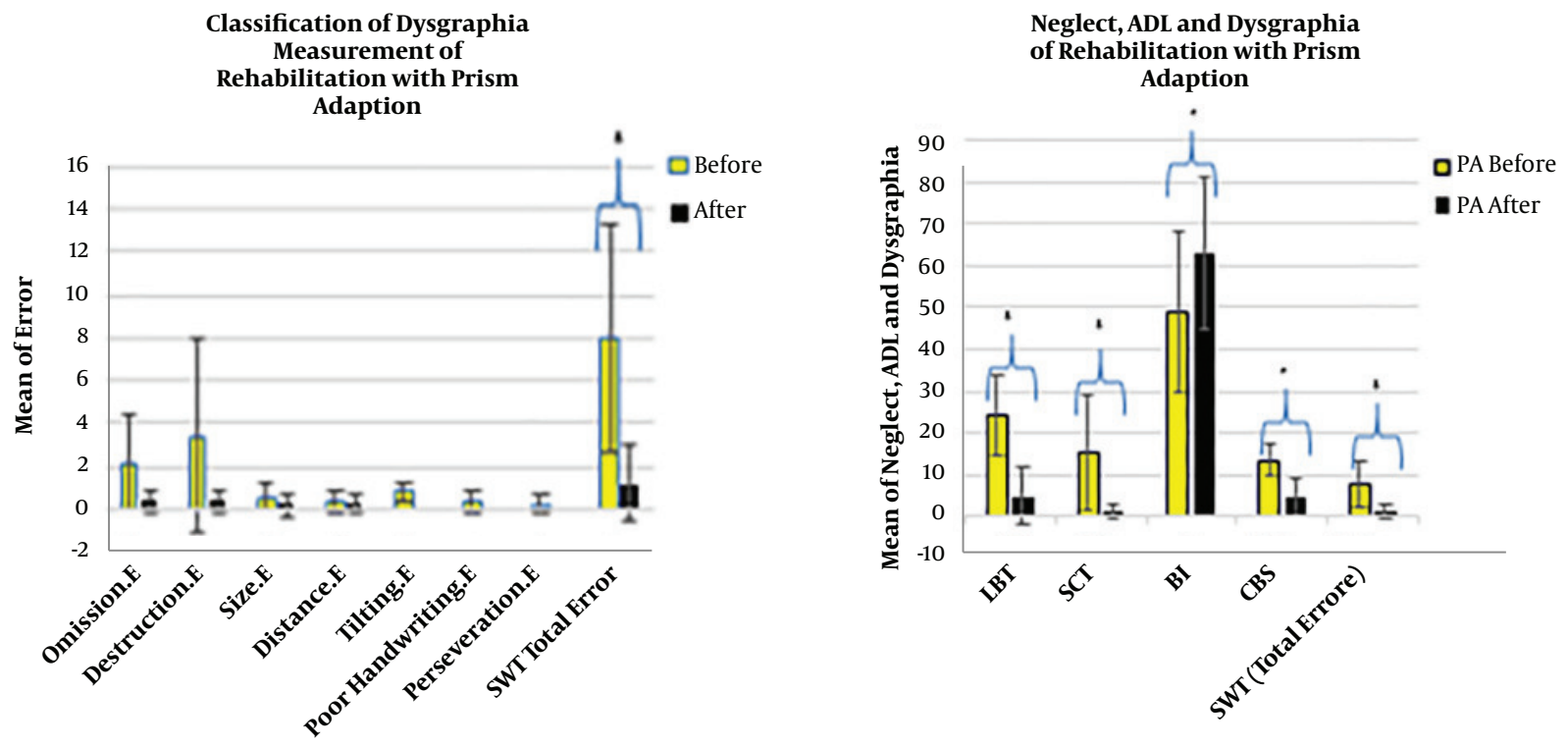

Figure 1. Figure on the left side shows patients with classification of dysgraphia in the spontaneous writing test of rehabilitation without writing practice before and after 10 sessions with PA. The Figure on the right side shows stroke patients' scores in LBT, SCT, BI, CBS and spontaneous writing test (total error) of rehabilitation without writing practice before and after 10 sessions with PA. Error bars indicate standard deviation. Asterisks indicate results that were significant using Wilcoxon tests: ${ }^{* *} \mathrm{P}<0.05$.

In the $\mathrm{BI}$ test, in this questionnaire, patients are queried about their independence in the activities of daily function such as feeding, urine or bowel incontinence, toilet use, dressing, and grooming. Its scores range from zero to 100 with higher scores denoting more independence in the ADL.

In the CBS test consists of 10 items related to real life conditions, such as grooming, dressing, eating, mouth cleaning, gaze orientation, knowledge of left limbs, auditory attention, moving, spatial orientation, and finding personal belongings. The items are scored ranging from 0 to 3 ; the total score of the scale was 33 . The CBS scale was divided in the two sub-scores evaluating the items relative to the visual search attentional items: 1, 3, 4, 5, 7,10, and to the 
motor-exploratory items: 2, 6, 8, 9, as proposed by Goedert et al. (20).

\subsection{PA Intervention}

The prism glasses with 10 degrees of the visual field displacement to right shift with adaption mirror were given to the patients for 20 minutes. The patients sat near a table on which a mirror box $(35 \times 35 \times 30 \mathrm{~cm})$ was placed vertically. They observed the reflection of the right intact hand as the movement of the left hand in the mirror. Therefore, the PA group underwent sham magnetic stimulation by tilting the coil vertically, which resulted in patients hearing magnetic pulse sounds without any stimulation. The patients received 10 sessions of rehabilitation for 2 weeks. Participants were blind to the type of brain stimulation therapy they received.

\subsection{Statistical Analysis}

We used SPSS version 24. The Kolmogorov-Smirnov normality test was performed and the Wilcoxon test was applied to compare outcome measure score before and after the intervention. The significance level was set at $\mathrm{P}<0.05$.

\section{Results}

\subsection{Writing and Rehabilitation}

All patients showed improvement in dysgraphia (total error score of spontaneous writing test) as revealed by Wilcoxon test $(\mathrm{P}=0.043)$. Figure 1 illustrates the mean spontaneous writing test scores at start and finish of the 10 sessions of intervention with PA.

Dysgraphia in spontaneous writing test was in preintervention with a total errors score of 40 as revealed by sum of score of classification of errors in the spontaneous writing test. Post intervention showed decrease of total errors score to 6. Destruction error was the most common error and the least prevalent was the omission writing error followed by patient with dysgraphia. A decrease was observed in the scores of all classifications of dysgraphia which suggests recovery in dysgraphia symptoms in prism adaption group after rehabilitation (Figure 1).

Classification of dysgraphia in the spontaneous writing test, visuospatial omission, visuospatial destruction errors, visuospatial tilting errors, poor handwriting, visuospatial distance errors, visuospatial perseveration (addition) and visuospatial size errors were detected to sum of score of errors in the participant before and after rehabilitation (examples shown in Figures 1 and 2).

Dysgraphia recovered to $85 \%$ of the maximum impairment measurable by the spontaneous writing test in PA group after rehabilitation.
4.2. Unilateral Spatial Neglect and Neurological Functional Activities of Rehabilitation

The secondary outcome in neglect symptoms and ADL were change in the SCT $(\mathrm{P}=0.043)$, LBT $(0.043)$, CBS (0.042) and BI (0.042) scores. All participants showed recovery in neglect and ADL after 10 sessions of rehabilitation with prism adaption. The Wilcoxon test showed a significant difference in LBT, SCT, CBS and BI scores after rehabilitation with $\mathrm{PA}(\mathrm{P}<0.05)$ (examples shown in Figure 1). The results of significant improvement in the neglect and ADL and the means of scores are summarized in

\section{Discussion}

The pilot study showed that, in all stroke patients with dysgraphia, neglect and ADL were improved after 10 sessions of rehabilitation with prism adaption. Dysgraphia, constructive errors of writing, were assessed in Persian patients with right-brain stroke. Dysgraphia was classified into visuospatial omission, visuospatial destruction, visuospatial tilting, visuospatial poor handwriting, visuospatial distance, visuospatial perseveration, and visuospatial size errors. Classification of dysgraphia were identified in Persian right-brain stroke patients' neglect. The most and least frequent errors were visuospatial destruction errors, and visuospatial omission errors, respectively.

Over $14 \%$ of the patients with pure superficial posterior cerebral artery territory infarction in clinical presentation suffer from dysgraphia due to stroke-induced neglect. It is mostly associated with dysgraphia, neglect induced cognitive impairment, and neurological dysfunction $(5,23)$. Writing is a human communication tool. However, there is limited research into therapy for adults with acquired dysgraphia. Non-pharmacological approaches have been shown to aid rehabilitation in dysgraphia. Non-pharmacological treatments used include spelling therapy, PA, RTMS, relearning in based spelling therapies on functional writing with unity-modal and multi-modal therapies, technology-enhanced writing therapy using assistive writing software to improve email writing, and rereading treatment with slow reading coupled interactive spelling treatment with errorful spelling $(8,12,13,15,24-27)$. The novelty of the present study was without writing practice, a new suggestion was provided for dysgraphia pattern and rehabilitation of dysgraphia, neglect and disability function.

In contrast, our present study of without practice in writing of recovery, previous studies on the dysgraphia therapy methods are based on writing, copying, spelling, and learning of practices in writing $(12,15)$. Inhibition of 

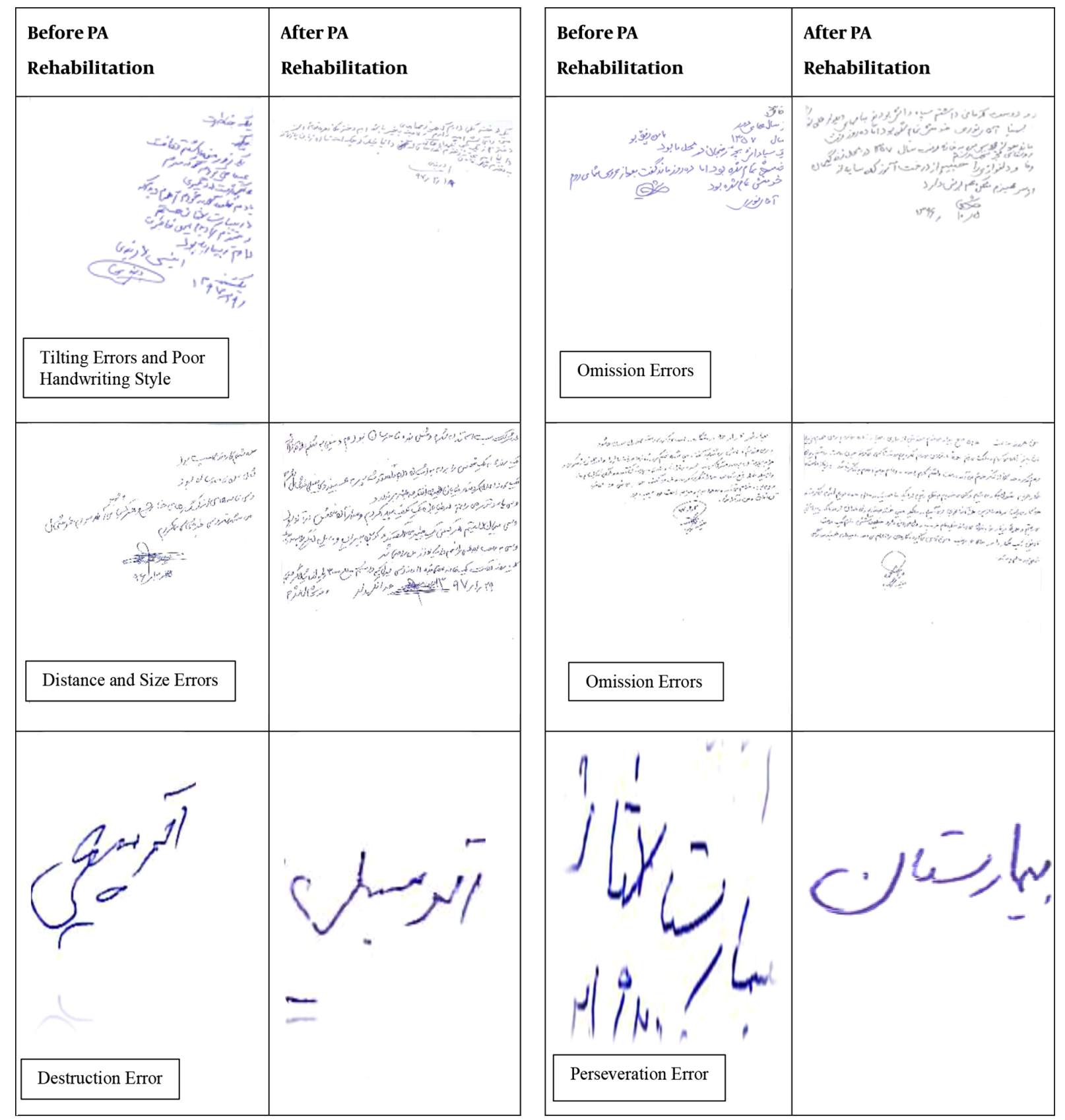

Figure 2. The classification of dysgraphia or constructive errors of writing in the Persian language was used in this study. The Figure on the left side of columns shows examples of the response of the patients with dysgraphia and neglect before intervention. Those on the right side of columns the response of the patients with dysgraphia and neglect of rehabilitation after 10 sessions without writing practice.

left parietal cortex in prism adaptation, the improvement of neglect after treatment, promotion of frontoparietal attention network in PA of mechanism may underlie rehabilitation effect seen in stroke-induced dysgraphia, neglect and disability function in our study (27-29).
In contrast, prism adaption approach has shown to treat neglect symptoms and ability of function in stroke patients with variable success. Prism adaption for rehabilitation, was useful in the neglect and activities of motor function rehabilitation in some clinical trials $(30,31)$, and 
not useful in some others $(32,33)$. In a recent study, prism adaptation was useful for both dysgraphia, and neglect symptoms (8). In the current study, patients attended rehabilitation sessions of PA for dysgraphia, neglect symptoms and activity of daily living, conformed to previous studies using therapeutic approaches such as PA designed to improve stroke-related spatial neglect and dysgraphia symptoms. The patient received PA wedge lenses creating an optical right shift of $10^{\circ}$. Line bisection, line cancellation, free drawing, and writing tasks were conducted one day before, and 2 hours before rehabilitation, after the short PA session, at baseline, and 2 hours after, 24 hours after, and 48 hours after rehabilitation. Dysgraphia was omissions error, duplications (addition) error, letters or words of abnormal spaces error. In single case, in a 69-year-old, righthanded man, improvement was observed in dysgraphia and the leftward orienting behavioral biases of the neglect syndrome following right-brain damage (8).

Classification of dysgraphia patterns in the present study findings showed that visuospatial destruction errors and omission errors were the most common subtype of constructive errors of writing in Persian patients with visuospatial unilateral neglect.

Destruction error outcome was consistent with studies on Japanese. Japanese participants demonstrated disorder related to ideogram Kanji writing by a top to bottom, vertical writing (3), Spanish patients' destruction error was measured by make-up word, disorganized and destroying (4), and Korean patients $(1,9,10)$.

Omission outcome was consistent with a study on Korean patients (10). These findings were in accordance with that of previous studies that showed left space omission, these constructive errors of writing have been reported in people of various languages such as Spanish (4), French (8), Japanese (3), and Korean (10). In contrast, previous studies including that of Jang et al. have reported right space omission (9); in the Iranian graphic system an onset always begins from right to left and in Korean it begins with a consonant on the left side. Korean people have been schooled to retain the stringently defined location for each grapheme within a syllabic square. Accordingly, regardless of left side neglect, Korean patients with neglect try to keep the left margin exaggeratedly starting the writing due to neglect related stroke. Ignoring the right space pattern of error could be specific to the Korean language. In contrast, previous studies including that of Caramazza and Hillis have reported right space omission. Left handed, neglect due to a stroke involving left basal ganglia and left parietal cortex of damage mechanisms may underlie right space omission effect seen in dysgraphia with right space omission (7).
Addition (perseveration) outcome was consistent with studies on Spanish in Roman alphabetic systems, Korean Han-Geul letters $(4,9,10)$. Omission and duplication (perseveration) error were mostly common subtypes of constructive errors of dysgraphia in Japanese patients, and about two-third had frontal lobe damage related stroke (3). Addition patterns were represented by Kanji, Chinese characters, Kana, and Han-Geul letters structurally analogous in their a top to bottom vertical organization as well as from left to right horizontal error on any part of the grapheme $(3,9,10)$.

Brain processing of the distance estimate and size estimate has been disturbed in patients with neglect related stroke, and were observed subsequently in writing of distance error, size of error, poor handwriting, and tilting error in patients with stroke-induced neglect (23). Tilting error showed to right cortex stroke $(1,10)$. Prism adaption method recovered visual deficit filed. Rehabilitation of neglect mechanisms may underlie improvement effect seen in dysgraphia in the Persian patients' written system to treat of errors in tilting, distance, size, and poor hand writing style in present study. This outcome of before recovery was consistent with studies languages such as Spanish (4), French (8), and Korean (1).

The pilot study had some limitations. Firstly, only a single center was assessed, not followed up and the study had a relatively small sample size. Secondly, only single characters were used for the writing tests. In addition, $40 \%$ of our patients were in the sub-acute phase below 6 months. PA is a safe and inexpensive approach without practice in writing; therefore, it is a potential home-based rehabilitation method.

Further studies on the relationship between dysgraphia and the underlying impaired mechanisms of the brain using neuroimaging are required.

In the present study, an influence was found between dysgraphia patterns in the Persian patients' written system and neglect in brain lesions related to stroke. Dysgraphia patterns of Persian language in patients were increased distance error, increased size error, omission error, destruction error, tilting error, poor handwriting style, and perseveration error. The present study findings showed that dysgraphia and neglect may be affected by recovery and educational strategies. In addition, unilateral visuospatial neglect appears to specifically affect the written system of Persian patients with right-brain stroke. The PA method could be a potentially useful tool for rehabilitating stroke patients with dysgraphia, neglect and ADL. 


\section{Acknowledgments}

We thank National Brain Mapping Lab (NBML).

\section{Footnotes}

Authors' Contribution: Study concept and design: Shole Vatanparasti and Ali Yoonessi. Analysis and interpretation of data: Anoshirvan Kazemnejad, Shole Vatanparasti and Ali Yoonessi. Drafting of the manuscript: Shole Vatanparasti. Critical revision of the manuscript: Ali Yoonessi. Study supervision: Shahram Oveisgharan and Ali Yoonessi. Statistical analysis: Anoshirvan Kazemnejad and Shole Vatanparasti.

Conflict of Interests: No conflict of interests is reported. Ethical Approval: The study was approved by the Ethics Committee of Iran University of Medical Sciences. This study was registered at https:/|fa.irct.ir/login Iranian Registry of Clinical Trials [IRCT20170423033606N3].

Funding/Support: This work was supported by the Iran Cognitive Sciences and Technologies Council (grant no.1446508).

Patient Consent: All the subjects gave their informed consents before conducting the study [IR.IUMS.REC.1396.93012334].

\section{References}

1. Lee M, Suh MK, Lee MH, Lee JS, Moon SY. Agraphia caused by acute right parietal infarction. J Clin Neurosci. 2015;22(4):758-60. doi: 10.1016/j.jocn.2014.09.023. [PubMed: 25564267].

2. Berlucchi G, Vallar G. The history of the neurophysiology and neurology of the parietal lobe. Handb Clin Neurol. 2018;151:3-30. doi: 10.1016/B978-0-444-63622-5.00001-2. [PubMed: 29519464].

3. Seki K, Ishiai S, Koyama Y, Sato S, Hirabayashi H, Inaki K, et al. Effects of unilateral spatial neglect on spatial agraphia of kana and kanji letters. Brain Lang. 1998;63(2):256-75. doi: 10.1006/brln.1997.1944. [PubMed: 9654434].

4. Ardila A, Rosselli M. Spatial agraphia. Brain Cogn. 1993;22(2):137-47. doi:10.1006/brcg.1993.1029. [PubMed: 8373568].

5. Cals N, Devuyst G, Afsar N, Karapanayiotides T, Bogousslavsky J. Pure superficial posterior cerebral artery territory infarction in The Lausanne Stroke Registry. J Neurol. 2002;249(7):855-61. doi: 10.1007/s00415-002-0742-0. [PubMed: 12140669].

6. Danna J, Velay JL, Paz-Villagrán V, Capel A, Petroz C, Gondre C, et al. Handwriting movement sonification for the rehabilitation of dysgraphia. 10th International Symposium on Computer Music Multidisciplinary Research (CMMR)-Sound, Music \& Motion-15-18 oct. 2013-Marseille. France. 2013. p. 200-8.

7. Caramazza A, Hillis AE. Spatial representation of words in the brain implied by studies of a unilateral neglect patient. Nature. 1990;346(6281):267-9. doi: 10.1038/346267a0. [PubMed: 2374591]

8. Rode G, Pisella L, Marsal L, Mercier S, Rossetti Y, Boisson D. Prism adaptation improves spatial dysgraphia following right brain damage. Neuropsychologia. 2006;44(12):2487-93. doi: 10.1016/j.neuropsychologia.2006.04.002. [PubMed: 16712882].
9. Jang DH, Kim MW, Park KH, Lee JW. Language-specific dysgraphia in Korean patients with right brain stroke: Influence of unilateral spatial neglect. J Korean Med Sci. 2015;30(3):323-7. doi: 10.3346/jkms.2015.30.3.323. [PubMed: 25729257]. [PubMed Central: PMC4330489].

10. Yoon JH, Suh MK, Kim H. Language-specific dysgraphia in Korean stroke patients. Cogn Behav Neurol. 2010;23(4):247-55. doi: 10.1097/WNN.0b013e3181c2955e. [PubMed: 21150348].

11. Cubelli R. A selective deficit for writing vowels in acquired dysgraphia. Nature. 1991;353(6341):258-60. doi: 10.1038/353258a0. [PubMed: 1896072].

12. Thiel L, Conroy P. A comparison of errorless and errorful therapies for dysgraphia after stroke. Neuropsychol Rehabil. 2014;24(2):172-201. doi: 10.1080/09602011.2013.875925. [PubMed: 24517315].

13. Thiel L, Sage K, Conroy P. Comparing uni-modal and multimodal therapies for improving writing in acquired dysgraphia after stroke. Neuropsychol Rehabil. 2016;26(3):345-73. doi: 10.1080/09602011.2015.1026357. [PubMed: 25854414].

14. Marshall J, Caute A, Chadd K, Cruice M, Monnelly K, Wilson S, et al. Technology-enhanced writing therapy for people with aphasia: Results of a quasi-randomized waitlist controlled study. Int J Lang Commun Disord. 2019;54(2):203-20. doi: 10.1111/1460-6984.12391. [PubMed: 29749112].

15. Thiel L, Sage K, Conroy P. The role of learning in improving functional writing in stroke aphasia. Disabil Rehabil. 2016;38(21):2122-34. doi: 10.3109/09638288.2015.1114038. [PubMed: 26727994].

16. Halligan PW, Marshall JC, Wade DT. Visuospatial neglect: Underlying factors and test sensitivity. Lancet. 1989;2(8668):908-11. doi: 10.1016/s0140-6736(89)91561-4. [PubMed: 2571823].

17. Guariglia P, Matano A, Piccardi L. Bisecting or not bisecting: This is the neglect question. Line bisection performance in the diagnosis of neglect in right brain-damaged patients. PLoS One. 2014;9(6). e99700. doi: 10.1371/journal.pone.0099700. [PubMed: 24937472]. [PubMed Central: PMC4061067].

18. Bonato M, Priftis K, Marenzi R, Zorzi M. Modulation of hemispatial neglect by directional and numerical cues in the line bisection task. Neuropsychologia. 2008;46(2):426-33. doi: 10.1016/j.neuropsychologia.2007.08.019. [PubMed: 17931670].

19. Oveisgharan S, Shirani S, Ghorbani A, Soltanzade A, Baghaei A, Hosseini S, et al. Barthel index in a Middle-East country: Translation, validity and reliability. Cerebrovasc Dis. 2006;22(5-6):350-4. doi: 10.1159/000094850. [PubMed: 16888374].

20. Goedert KM, Chen P, Botticello A, Masmela JR, Adler U, Barrett AM. Psychometric evaluation of neglect assessment reveals motor-exploratory predictor of functional disability in acutestage spatial neglect. Arch Phys Med Rehabil. 2012;93(1):137-42. doi: 10.1016/j.apmr.2011.06.036. [PubMed: 22200393]. [PubMed Central: PMC3246635].

21. Azouvi P, Olivier S, de Montety G, Samuel C, Louis-Dreyfus A, Tesio L. Behavioral assessment of unilateral neglect: Study of the psychometric properties of the Catherine Bergego Scale. Arch Phys Med Rehabil. 2003;84(1):51-7. doi: 10.1053/apmr.2003.50062. [PubMed: 12589620].

22. Azouvi P, Jacquin-Courtois S, Luaute J. Rehabilitation of unilateral neglect: Evidence-based medicine. Ann Phys Rehabil Med. 2017;60(3):1917. doi: 10.1016/j.rehab.2016.10.006. [PubMed: 27986428].

23. Rode G, Pagliari C, Huchon L, Rossetti Y, Pisella L. Semiology of neglect: An update. Ann Phys Rehabil Med. 2017;60(3):177-85. doi: 10.1016/j.rehab.2016.03.003. [PubMed: 27103056].

24. Kakuda W, Abo M, Momosaki R, Morooka A. Therapeutic application of 6-Hz-primed low-frequency rTMS combined with intensive speech therapy for post-stroke aphasia. Brain Inj. 2011;25(12):1242-8. doi: 10.3109/02699052.2011.608212. [PubMed: 21902549] 
25. Thiel L, Sage K, Conroy P. Promoting linguistic complexity, greater message length and ease of engagement in email writing in people with aphasia: Initial evidence from a study utilizing assistive writing software. Int J Lang Commun Disord. 2017;52(1):106-24. doi: 10.1111/14606984.12261. [PubMed: 27297074].

26. Kim ES, Rising K, Rapcsak SZ, Beeson PM. Treatment for alexia with agraphia following left ventral occipito-temporal damage: Strengthening orthographic representations common to reading and spelling. J Speech Lang Hear Res. 2015;58(5):1521-37. doi: 10.1044/2015_JSLHR-L-14-0286. [PubMed: 26110814]. [PubMed Central: PMC4686312].

27. DeMarco AT, Wilson SM, Rising K, Rapcsak SZ, Beeson PM. The neural substrates of improved phonological processing following successful treatment in a case of phonological alexia and agraphia. Neurocase. 2018;24(1):31-40. doi: 10.1080/13554794.2018.1428352. [PubMed: 29350575]. [PubMed Central: PMC5843561].

28. Tsujimoto K, Mizuno K, Nishida D, Tahara M, Yamada E, Shindo $\mathrm{S}$, et al. Correlation between changes in functional connectivity in the dorsal attention network and the after-effects induced by prism adaptation in healthy humans: A dataset of resting-state fMRI and pointing after prism adaptation. Data Brief. 2019;22:583-9. doi: 10.1016/j.dib.2018.12.053. [PubMed: 30627613]. [PubMed Central: PMC6322076].

29. Martin-Arevalo E, Schintu S, Farne A, Pisella L, Reilly KT. Adaptation to leftward shifting prisms alters motor interhemispheric inhibition. Cereb Cortex. 2018;28(2):528-37. doi:10.1093/cercor/bhw386. [PubMed: 27993820]. [PubMed Central: PMC6248503].

30. Goedert KM, Chen P, Boston RC, Foundas AL, Barrett AM. Presence of motor-intentional aiming deficit predicts functional improvement of spatial neglect with prism adaptation. Neurorehabil Neural Repair. 2014;28(5):483-93. doi: 10.1177/1545968313516872. [PubMed: 24376064]. [PubMed Central: PMC4074266].

31. Hreha K, Gillen G, Noce N, Nilsen D. The feasibility and effectiveness of using prism adaptation to treat motor and spatial dysfunction in stroke survivors with multiple incidents of stroke. Top Stroke Rehabil. 2018;25(4):305-11. doi: 10.1080/10749357.2018.1437937. [PubMed: 29458299].

32. Turton AJ, O'Leary K, Gabb J, Woodward R, Gilchrist ID. A single blinded randomised controlled pilot trial of prism adaptation for improving self-care in stroke patients with neglect. Neuropsychol Rehabil.2010;20(2):180-96. doi:10.1080/09602010903040683. [PubMed: 19629848].

33. Ten Brink AF, Visser-Meily JMA, Schut MJ, Kouwenhoven M, Eijsackers ALH, Nijboer TCW. Prism adaptation in rehabilitation? No additional effects of prism adaptation on neglect recovery in the subacute phase poststroke: A randomized controlled trial. Neurorehabil Neural Repair. 2017;31(12):1017-28. doi: 10.1177/1545968317744277. [PubMed: 29192535]. 